\title{
Attitude Stabilization of Rigid Spacecraft with Minimal Attitude Coordinates and Unknown Time-varying Delay
}

\author{
Ehsan Samiei, Eric A. Butcher, Amit K. Sanyal and Robert Paz
}

\begin{abstract}
The delayed feedback stabilization of rigid spacecraft attitude dynamics in the presence of an unknown time-varying delay in the measurement is addressed. The attitude representation is parameterized using minimal attitude coordinates. The time-varying delay and its derivative are assumed to be bounded. By employing a linear state feedback controller via a Lyapunov-Krasovskii functional, a general delaydependent stability condition is characterized for the closed-loop parameterized system in terms of a linear matrix inequality(LMI) whose solution gives the suitable controller gains. An estimate of the region of attraction of the controlled system is also obtained, inside which the asymptotic stability of parameterized system is guaranteed.
\end{abstract}

\section{Index Terms}

attitude dynamics, linear matrix inequality, Lyapunov-Krasovskii functional, time-delay, region of attraction.

\section{INTRODUCTION}

Feedback stabilization of rigid body attitude dynamics is an important control problem, see e.g. [1]-[5], with a wide range of applications such as spacecraft attitude maneuvers [6], [7],

This work is supported by the National Science Foundation under Grant No. CMMI-1131646.

E. Samiei (corresponding author) and A.K. Sanyal are with the Department of Mechanical and Aerospace Engineering, New Mexico State University, Las Cruces, NM, 88003 USA. Tel: (575) 646-4319 (email:esamiei@nmsu.edu;asanyal@nmsu.edu).

E. A. Butcher is with the Department of Aerospace and Mechanical Engineering, University of Arizona, Tucson, AZ 85721 (e-mail:ebutcher@email.arizona.edu)

R. Paz is with the Department of Electrical and Computer Engineering, New Mexico State University, Las Cruces, NM 88003, USA. Tel: (575) 646-4933 (e-mail:rpaz@nmsu.edu). 
underwater vehicles [8], and robotic manipulators [9]. The attitude representation depends on the choice of attitude parameters used to represent the orientation of a rigid body relative to an inertial frame see, e.g. [10]-[12]. Several control laws have been developed for the control of rigid body attitude dynamics. In [13], [14], geometric controllers are designed on $\mathrm{SO}(3)$, which is the set of special orthogonal matrices, in order to track attitude and angular velocity commands while guaranteeing almost global asymptotic stability. In [3] an optimal controller is used, based on minimal attitude coordinates, to minimize a quadratic cost function for a dynamical system. Tracking control of a rigid asymmetric spacecraft is addressed in [7] by using a Hamiltonian-Jacobi formulation.

On the other hand, in several practical applications, there is an unavoidable time delay within the control system due to delay in measurements or actuators. Controlled systems designed based on feedback schemes are usually robust to a small amount of time delay. However, if the time delay increases due to the failure of system components or external sources, then the effect of this large time delay on the undesirable behavior of the controlled attitude motion is notable and may lead the non-delayed-based controlled system to chatter or produce oscillatory motion [15], [16]. However, to the authors' knowledge, there are few studies on delayed feedback control of attitude dynamics [15], [17]-[21]. In [20] a nonlinear robust controller is implemented without angular velocity measurements in the presence of a constant time delay in the control signal. The closed-loop system is shown to be stable for a norm bounded nonlinear uncertainty in the attitude dynamics. In [15], a velocity free output-based controller for attitude regulation of a rigid spacecraft considering the effects of a known time delay in the system is investigated. Sufficient conditions for attitude stabilization of the spacecraft are also obtained based on previously established controllers for manipulators. However, the asymptotic stability of system is only guaranteed for a sufficiently small time delay, while only a small estimate of the region of attraction was obtained. This conservatism has been fairly addressed in [21] by employing a linear state feedback controller for the attitude motion with an unknown time delay, which has a known upper bound in the feedback path using a frequency domain approach. A complete type Lyapunov-Krasovskii functional is constructed to ensure the robust stability of the linear controller, and an estimate of the region of attraction is obtained.

In this paper, the delayed feedback control of rigid spacecraft attitude dynamics is studied. Kinematic differential equations of the spacecraft are modeled using minimal attitude coordi- 
nates that can include well-known attitude parameters such as Euler angles, classical Rodriguez parameters (CRPs), modified Rodriguez parameters (MRPs) and exponential coordinates. We assume that there is an unknown time varying delay in the measurement (as opposed to actuator delay [17], [18]) with known upper bounds for both the delay and its rate. Unlike in [21], the controller gain matrices for the linear delayed feedback control law are obtained using a Lyapunov-Krasovskii functional in terms of a linear matrix inequality (LMI) which guarantees local asymptotic stability of the parameterized system. To cope with the nonlinear term in the dynamical model, we assume that the nonlinearities satisfy a nonlinear growth condition. Furthermore, an estimate of the region of attraction of the system is also obtained. Finally, a set of simulations is performed for a given set of spacecraft parameters.

This paper is organized as follows: In Section II, we present the attitude kinematics and dynamics model. Section III presents the delayed feedback controller design, while an estimate of the region of the attraction of the system is obtained in Section IV. Numerical simulation results are shown in Section V, and Section VI concludes the paper.

\section{SpaceCRAfT Attitude Dynamics}

In this section, we introduce some preliminary concepts of a minimal attitude parameterization $\xi \in \mathbb{R}^{3}$, and then introduce the kinematic and kinetic differential equations of a rigid spacecraft. Two coordinate frames in the three-dimensional Euclidean space are employed. $\mathcal{N}$ defines the inertial coordinate frame and $\mathcal{B}$ defines the body-fixed coordinate frame. The spacecraft is modeled as a rigid body. In addition, we suppose that there are three actuators acting along orthogonal axes in the frame $\mathcal{B}$. In general, the equations of motion obtained by using a minimal set of attitude coordinates $\xi \in \mathbb{R}^{3}$ can be expressed as

$$
\begin{aligned}
\dot{\xi}(t) & =\frac{1}{\beta}\left(\mathfrak{G}(\xi(t))+I_{3}\right) \omega(t), \\
J \dot{\omega}(t) & =-\omega(t)^{\times} J \omega(t)+u(t),
\end{aligned}
$$

where $\mathfrak{G}: \mathbb{R}^{3} \rightarrow \mathbb{R}^{3 \times 3}$ is a nonlinear function of $\xi, \beta$ is a constant scalar, which is selected according to the choice of attitude coordinates, $\omega(t) \in \mathbb{R}^{3}$ represents the angular velocity of the system described in $\mathcal{B}$ relative to $\mathcal{N}, J \in \mathbb{R}^{3 \times 3}$ is the known positive definite inertia matrix, 
$u(t) \in \mathbb{R}^{3}$ is the control torque input, $I_{3} \in \mathbb{R}^{3 \times 3}$ is the identity matrix, , and $(\cdot)^{\times}$is defined as

$$
\omega^{\times}=\left[\begin{array}{ccc}
0 & -\omega_{3} & \omega_{2} \\
\omega_{3} & 0 & -\omega_{1} \\
-\omega_{2} & \omega_{1} & 0
\end{array}\right] .
$$

Equation (1) is the kinematic differential equation, which can be considered as Euler angles, CRPs, MRPs, or exponential coordinates. Equation (1) is also analogous to Poisson's equation $\dot{C}(t)=-\omega(t)^{\times} C(t)$ where $C(t) \in \mathrm{SO}(3)$ is the direction cosine matrix that rotates vectors from $\mathcal{N}$ to $\mathcal{B}$, while the second part represents the rotational dynamics (Euler's equation).

Let define the state variable $x(t)=\left[x_{1}^{\mathrm{T}}(t), x_{2}^{\mathrm{T}}(t)\right]^{\mathrm{T}}=\left[\xi^{\mathrm{T}}(t),(1 / \beta) \omega^{\mathrm{T}}(t)\right]^{\mathrm{T}} \in \mathbb{R}^{6}$. Eq.(1) can be written as

$$
\dot{x}(t)=A x(t)+B u(t)+f(x(t))
$$

where

$$
\begin{aligned}
& A=\left[\begin{array}{cc}
0_{3 \times 3} & I_{3} \\
0_{3 \times 3} & 0_{3 \times 3}
\end{array}\right] \in \mathbb{R}^{6 \times 6}, B=\left[\begin{array}{c}
0_{3 \times 3} \\
(1 / \beta) J^{-1}
\end{array}\right] \in \mathbb{R}^{6 \times 3}, \\
& f(x(t))=\left[\begin{array}{l}
f_{1}(x(t)) \\
f_{2}(x(t))
\end{array}\right]=\left[\begin{array}{c}
\mathfrak{G}\left(x_{1}(t)\right) x_{2}(t) \\
-\beta J^{-1} x_{2}(t)^{\times} J x_{2}(t)
\end{array}\right] \in \mathbb{R}^{6},
\end{aligned}
$$

The equilibrium subspace of the attitude motion is obtained as $E=\left\{\left(x_{1}, x_{2}\right) \mid x_{1} \in \mathbb{R}^{3}, x_{2}=0\right\}$. The origin of the parameterized system, i.e, $x_{1}=x_{2}=0$ is considered as the desired equilibrium point of the system. We seek to design a controller for the attitude dynamics in the presence of time delay in the measurement such that the origin is asymptotically stable.

Any minimal attitude parameterizations contains at least one geometrical orientation where the attitude is singular, see e.g. [5], [11], [12]. For example, modified Rodriguez parameters (MRPs) denoted by $\sigma(t) \in \mathbb{R}^{3}$, have a singularity after one complete revolution. A singularityfree representation can be obtained if shadow set switching, which is an alternate set obtained from the projection of the other Euler parameters set, is performed [5]. However, switching is not employed in this study due to additional difficulties (e.g. chattering) resulting from discontinuous control in the presence of time delay. In addition, the exponential coordinates denoted by $\Theta \in \mathbb{R}^{3}$, which is a local diffeomorphism (non-singular) at the identity [22], [23] and is obtained from the exponential map contains an ambiguity when the spacecraft rotates by $\Phi=\pi \operatorname{rad}$ [24], where $\Phi$ is the principal rotation angle with the corresponding principal rotation axis $\hat{e}$. 


\section{Delayed FeedBack Controller Design}

The objective of controller design is to stabilize the parameterized spacecraft model of Eq. (3) such that all the angular velocities and attitude parameters go to zero as $t \rightarrow \infty$ in some region of the domain $\mathbb{R}^{6}$ that contains the origin in the presence of an unknown time-varying delay in the feedback path, i.e., $\lim _{t \rightarrow \infty}\left\{\|x(t)\|^{2}\right\}=0$. Thus, we design a linear controller for the nonlinear model of Eq.(3) by using the Lyapunov-Krasovskii method in conjunction with a LMI such that the local asymptotic stability of the solution is fulfilled. We assume that there is an unknown continuous time-varying time delay function $\tau(t) \in \mathbb{R}$ in the feedback loop such that

$$
0 \leq \tau(t) \leq \tau_{\max }, \quad \dot{\tau}(t) \leq d<1, \quad \forall t \geq 0,
$$

where $\tau_{\max }$ and $d$ are positive constant scalars. Thus, all the measured signals that go to the controller are driven by the time delay. In addition, there are no control torque constraints.

\section{A. Preliminary Results}

According to the above discussion, the linear state feedback control torque can be chosen as

$$
u(t)=J\left[\beta K_{1} \xi(t-\tau(t))+K_{2} \omega(t-\tau(t))\right]=K x_{\tau}
$$

where $K=\left[\begin{array}{lll}J \beta K_{1} & J K_{2}\end{array}\right] \in \mathbb{R}^{3 \times 6}$ and $K_{1}, K_{2} \in \mathbb{R}^{3 \times 3}$ are constant control gain matrices. We denote $x(t) \equiv x$ and $x(t-\tau(t)) \equiv x_{\tau}$. Substituting Eq.(6) into Eq.(3), the closed-loop system can be obtained in the form of a nonlinear delay-differential equation (DDE) as

$$
\begin{aligned}
& \dot{x}=A x+B K x_{\tau}+f(x) \\
& x(\theta)=\phi(\theta), \quad-\tau_{\max } \leq \theta \leq 0,
\end{aligned}
$$

where $\phi(\theta)$ is the initial function, the infinite dimensional state defined by $x_{t}(\theta)=x(t+$ $\theta),-\tau_{\max } \leq \theta \leq 0$ resides in the Banach space $\mathcal{C}\left(\left[-\tau_{\max }, 0\right], \mathbb{R}^{6}\right)$, which is the set of continuous functions mapping $\left[-\tau_{\max }, 0\right]$ to $\mathbb{R}^{6}$.

In this paper, we are interested in the stabilization problem of such systems in the presence of the time-varying delay in the measurement using the time domain approach. Therefore, a Lyapunov-based method such as Lyapunov-Krasovskii functional, which extends the standard Lyapunov stability method to delay differential equations, is employed. The following lemma 
provides the sufficient conditions for the stability analysis of attitude motion in the presence of time delay.

Lemma 1. (Lyapunov-Krasovskii)(see, E.g., [25], [26]): The trivial solution of Eq.(7) is asymptotically stable if there exists a scalar functional candidate $V\left(t, x_{t}(\theta)\right)$ such that

$$
u(\|x(t)\|) \leq V\left(t, x_{t}(\theta)\right) \leq v\left(\left\|x_{t}(\theta)\right\|_{m}\right),
$$

where $\|\cdot\|=\|\cdot\|_{2}$ represents the Euclidean norm and $\left\|x_{t}(\theta)\right\|_{m}=\sup \left\{\left\|x_{t}(\theta)\right\|\right\}$, and

$$
\dot{V}\left(t, x_{t}(\theta)\right) \leq-w(\|x(t)\|) \mathrm{d} t,
$$

where $u(s), v(s)$, and $w(s)>0$ for all $s>0$.

Fro the above Lemma, we set $w(s)=\delta s^{2}$ for some $\delta>0$.

The following Lemma is also considered.

Lemma 2. ([27]) For any given $a(t) \in \mathbb{R}^{n}, b(t) \in \mathbb{R}^{m}$, and $N \in \mathbb{R}^{n \times m}$ on the interval $\mathscr{L} \subset \mathbb{R}$, and any matrices $M \in \mathbb{R}^{n \times n}, Y \in \mathbb{R}^{n \times m}$, and $R \in \mathbb{R}^{m \times m}$, the inequality

$$
\begin{aligned}
& -2 \int_{\mathscr{L}} a^{T}(s) N b(s) \mathrm{d} s \leq \\
& \int_{\mathscr{L}}\left[\begin{array}{l}
a(s) \\
b(s)
\end{array}\right]^{T}\left[\begin{array}{cc}
M & Y-N \\
Y^{T}-N^{T} & R
\end{array}\right]\left[\begin{array}{l}
a(s) \\
b(s)
\end{array}\right] \mathrm{d} s,
\end{aligned}
$$

always holds, where

$$
\left[\begin{array}{cc}
M & Y \\
Y^{T} & R
\end{array}\right] \geq 0 .
$$

It is worth mentioning that the above inequality introduced in [27] is less conservative in comparison with other approaches for estimating the upper bounds of cross terms $-2 a^{T} b$. In the following, we use the above-mentioned Lemma for designing a delayed feedback controller in order to ensure that stability conditions and the estimate of the region of attraction of the rigid body attitude motion are less conservative. 
B. State Feedback Controller Design

Regarding the nonlinear vector $f(x)$, we have the following Lemma:

Lemma 3. The nonlinear function $f(x)$ defined in Eq.(3) satisfies $f(0)=0$ and can be rewritten as $f(x)=F(x) x$, where $F(x)$ is a smooth function and is obtained as

$$
F(x)=\left[\begin{array}{cc}
0_{3 \times 3} & \mathfrak{G}\left(x_{1}\right) \\
0_{3 \times 3} & -\beta J^{-1} x_{2}^{\times} J
\end{array}\right] \in \mathbb{R}^{6 \times 6} .
$$

Denote the induced 2-norm (see, i.e, [28]) of $F(x)$ by $\|F(x)\|=\gamma(x)$, where $\gamma(x)$ is a positive real-valued function. We consider the neighborhood $\mathscr{N} \subset \mathbb{R}^{6}$ of the origin such that $\mathscr{N}=\{x \in$ $\left.\mathbb{R}^{6}:\|x\| \leq k\right\}$, where $k>0$ is a known constant. Therefore $f(x)$ satisfies a bounded growth condition in $\mathbb{R}^{6}$ such that $\|f(x)\| \leq \gamma(x)\|x\| \leq \gamma(k)\|x\|$. In addition, there exists a positive constant $\gamma_{\max }$ such that $0 \leq \gamma(k) \leq \gamma_{\max }$.

The Proof of this Lemma will be discussed in Section IV. In the following theorem, we use the Lyapunov-Krasovskii functional method to derive a feasible LMI such that it determines the controller gain matrix $K$ to stabilize the closed-loop dynamical system in Eq.(7).

Theorem 1. There exists a linear state feedback controller in the form of Eq.(6) such that the solution of the closed-loop parameterized system in Eq.(7) is asymptotically stable for any given time delay $\tau(t)$ satisfying Eq.(5) and a certain range of $\gamma_{\max }$, if there exists positive scalars $k_{h}$ and symmetric matrices $X, W, Z, \bar{K}_{P}, K_{Z}>0$, matrices $G, V, H$ such that the following LMIs 
hold:

$$
\begin{aligned}
& \min \left(\operatorname{tr}\left(\bar{K}_{P}-K_{Z}\right)+k_{h}\right) \\
& \text { subject to } \\
\bar{\Psi}_{2}= & {\left[\begin{array}{cccc}
\Omega_{2} & \Gamma_{2} & \tau_{\max } X A^{T} & \tau_{\max } \gamma_{\max } X \\
\star & \Delta_{2} & \tau_{\max } H^{T} B^{T} & 0 \\
\star & \star & -\tau_{\max } Z & 0 \\
\star & \star & \star & -\tau_{\max } K_{Z}
\end{array}\right]<0, } \\
\Sigma= & {\left[\begin{array}{cc}
V & G \\
G^{T} & 2 X-Z
\end{array}\right] \geq 0, } \\
\Upsilon= & {\left[\begin{array}{cc}
\bar{K}_{P} & X \\
X & X
\end{array}\right] \geq 0, } \\
& {\left[\begin{array}{cc}
k_{h} I_{3 \times 3} & H^{T} \\
H & I_{3 \times 3}
\end{array}\right] \geq 0, \quad Z-K_{Z} \geq 0, }
\end{aligned}
$$

where $\star$ represents the terms that are induced by symmetry,

$$
\begin{aligned}
\Omega_{2}= & X A^{T}+A X+W+\tau_{\max } \bar{d} V \\
& +\bar{d}\left(G+G^{T}\right)+2 \gamma_{\max } \bar{K}_{P}, \\
\Gamma_{2}= & B H-\bar{d} G, \quad \Delta_{2}=-\bar{d} W
\end{aligned}
$$

and

$$
\begin{aligned}
& P=X^{-1}, Q=P W P, M=P V P, Y=P G P, \\
& R=Z^{-1}, K_{P}=P \bar{K}_{P} P .
\end{aligned}
$$

Then, the control gain matrix $K$ can be obtained as

$$
K=H X^{-1}=H P
$$

Proof. Define the Lyapunov-Krasovskii functional candidate $V\left(t, x_{t}(\theta)\right): \mathbb{R} \times \mathcal{C}\left(\left[-\tau_{\max }, 0\right], \mathbb{R}^{6}\right) \rightarrow$ $\mathbb{R}$ as

$$
\begin{aligned}
& V\left(t, x_{t}(\theta)\right)=x^{\mathrm{T}} P x+\int_{t-\tau(t)}^{t} x^{\mathrm{T}}(s) Q x(s) \mathrm{d} s \\
& +\int_{t-\tau(t)}^{t} \int_{\eta}^{t}\left[\zeta^{\mathrm{T}}(s) R \zeta(s)+f^{\mathrm{T}}(x(s)) R f(x(s))\right] \mathrm{d} s \mathrm{~d} \eta,
\end{aligned}
$$


where $P, Q$, and $R \in \mathbb{R}^{6 \times 6}$ are symmetric positive definite matrices, respectively, which will be determined later and $\zeta(t)=A x+B K x_{\tau}$. In addition, Let $P \leq K_{P}$ and $R \leq K_{R}$, where $K_{P}, K_{R} \in \mathbb{R}^{6 \times 6}$ are symmetric positive definite matrices. Equation (16) is positive in the Banach space $\mathcal{C}\left(\left[-\tau_{\max }, 0\right], \mathbb{R}^{6}\right)$ except at the origin and differentiable with respect to $x$ and $t$. Note that separating linear and nonlinear terms inside the integral term in the above proposed M-L-K functional leads to less nonlinear cross terms in the derivative of the M-L-K functional candidate in comparison with the standard L-K functional approach and as a result less conservative stability conditions can be obtained. Taking the time derivative of the above Lyapunov functional candidate along the trajectories of Eq.(7) and considering the properties of the time-varying delay described in Eq.(5) yields

$$
\begin{aligned}
& \dot{V}\left(t, x_{t}(\theta)\right) \leq 2 x^{\mathrm{T}} P\left(A x+B K x_{\tau}+f(x)\right)+x^{\mathrm{T}} Q x-\bar{d} x_{\tau}^{\mathrm{T}} \\
& \times Q x_{\tau}-\bar{d} \int_{t-\tau(t)}^{t}\left[\zeta^{\mathrm{T}}(s) R \zeta(s)+f^{\mathrm{T}}(x(s)) R f(x(s))\right] \mathrm{d} s \\
& +\int_{t-\tau(t)}^{t}\left[\zeta^{\mathrm{T}}(t) R \zeta(t)+f^{\mathrm{T}}(x(t)) R f(x(t))\right] \mathrm{d} s,
\end{aligned}
$$

where $\bar{d}=(1-d)$. On the other hand, applying the Newton-Leibniz formula to Eq.(7) yields $2 \bar{d} x^{\mathrm{T}}(t) E\left(x-x_{\tau}-\int_{t-\tau(t)}^{t}[\zeta(s)+f(x(s))] \mathrm{d} s\right)=0$, where $E \in \mathbb{R}^{6 \times 6}$ is any matrix. An upper-bound for the first integral term in the aboved-mentioned Newton-Leibniz formula can be obtained by employing Lemma 2 as

$$
\begin{aligned}
& -2 \bar{d} x^{\mathrm{T}} E \int_{t-\tau(t)}^{t} \zeta(s) \mathrm{d} s \leq \bar{d} \tau_{\max } x^{\mathrm{T}} M x+2 \bar{d} x^{\mathrm{T}}(Y-E) \\
& \int_{t-\tau(t)}^{t} \zeta(s) \mathrm{d} s+\bar{d} \int_{t-\tau(t)}^{t} \zeta^{\mathrm{T}}(s) R \zeta(s) \mathrm{d} s
\end{aligned}
$$

where the matrix inequality $\Sigma \geq 0$ with the elements $\Sigma_{(1,1)}=M, \Sigma_{(1,2)}=Y, \Sigma_{(2,1)}=$ $Y^{\mathrm{T}}$, and $\Sigma_{(2,2)}=R$ as a result of using Lemma 2 is satisfied. Similarly, the integral term $-2 \bar{d} x^{\mathrm{T}} E \int_{t-\tau(t)}^{t} f(x(s)) \mathrm{d} s$ from the aboved-mentioned Newton-Leibniz formula can be upperbounded by using Lemma 2 as

$$
\begin{aligned}
& -2 \bar{d} x^{\mathrm{T}} E \int_{t-\tau(t)}^{t} f(x(s)) \mathrm{d} s \leq \bar{d} \tau_{\max } x^{\mathrm{T}} M_{1} x+2 \bar{d} x^{\mathrm{T}}(Y-E) \\
& \int_{t-\tau(t)}^{t} f(s) \mathrm{d} s+\bar{d} \int_{t-\tau(t)}^{t} f^{\mathrm{T}}(s) R f(s) \mathrm{d} s
\end{aligned}
$$


where the matrix inequality $\Sigma_{1} \geq 0$ with the elements $\Sigma_{1_{(1,1)}}=M_{1}, \Sigma_{1_{(1,2)}}=Y, \Sigma_{1_{(2,1)}}=Y^{\mathrm{T}}$, and $\Sigma_{1_{(2,2)}}=R$ holds. Inserting the upper-bounds of Eqs. (18) and (19) into $\mathrm{Eq}(17)$ yields

$$
\begin{aligned}
& \dot{V}\left(t, x_{t}(\theta)\right) \leq x^{\mathrm{T}}\left(P A+A^{\mathrm{T}} P+Q+\tau_{\text {max }} A^{\mathrm{T}} R A\right. \\
& \left.+\tau_{\text {max }} \bar{d}\left(M+M_{1}\right)+\bar{d}\left(Y+Y^{\mathrm{T}}\right)\right) x+2 x^{\mathrm{T}}(P B K \\
& \left.+\tau_{\max } A^{\mathrm{T}} R B K-\bar{d} Y\right) x_{\tau}+x_{\tau}^{\mathrm{T}}\left(-\bar{d} Q+\tau_{\max } K^{\mathrm{T}} B^{\mathrm{T}}\right. \\
& \times R B K) x_{\tau}+2 x^{\mathrm{T}} P f(x)+\tau_{\text {max }} f(x)^{\mathrm{T}} R f(x) .
\end{aligned}
$$

Considering Lemma 3 and the defined inequalities $P \leq K_{P}$ and $R \leq K_{R}$, the time derivative of the L-K functional in the matrix form is obtained as

$$
\dot{V}\left(t, x_{t}(\theta)\right) \leq \bar{x}^{\mathrm{T}}(t) \Psi \bar{x}(t),
$$

where $\bar{x}(t)=\left[x(t)^{\mathrm{T}} x^{\mathrm{T}}(t-\tau(t))\right]^{\mathrm{T}}$ is the new state variable and matrix $\Psi$ is defined as

$$
\Psi=\left[\begin{array}{c}
\Omega \Gamma \\
\Gamma^{\mathrm{T}} \Delta
\end{array}\right],
$$

and

$$
\begin{aligned}
& \Omega=P A+A^{\mathrm{T}} P+Q+\tau_{\max } A^{\mathrm{T}} R A+\tau_{\max } \bar{d}\left(M+M_{1}\right) \\
& +\bar{d}\left(Y+Y^{\mathrm{T}}\right)+2 K_{P} \gamma_{\max }+\tau_{\max } \gamma_{\max }^{2} K_{R}, \\
& \Gamma=P B K+\tau_{\max } A^{\mathrm{T}} R B K-\bar{d} Y, \\
& \Delta=-\bar{d} Q+\tau_{\max } K^{\mathrm{T}} B^{\mathrm{T}} R B K .
\end{aligned}
$$

Therefore, based on the definition of the Lyapunov-Krasovskii functional in Lemma 1, the stability of Eq.(7) is guaranteed if Eq.(21) is satisfied such that

$$
\dot{V}\left(t, x_{t}(\theta)\right) \leq x^{\mathrm{T}}(t) \Psi \bar{x}(t) \leq-\delta\|x(t)\|^{2} .
$$

In addition, matrix inequities $\Sigma, \Sigma_{1} \geq 0, P \leq K_{P}$, and $R \leq K_{R}$ hold. We combine two matrix inequalities $\Sigma, \Sigma_{1} \geq 0$, which are concluded as a result of employing Lemma 2 for two integral terms in Eqs.(18) and (19), to obtain $\Sigma_{2} \geq 0$, where $\Sigma_{2_{(1,1)}}=M, \Sigma_{2_{(1,2)}}=Y, \Sigma_{2_{(2,1)}}=Y^{\mathrm{T}}$, and $\Sigma_{2_{(2,2)}}=R$, and $M=M_{1}+M_{2}$. Furthermore, the matrix inequality $P \leq K_{P}$ can be represented in an equivalent matrix form by employing the Schur complement as $\Upsilon>0$ with the elements 
$\Upsilon_{(1,1)}=K_{P}, \Upsilon_{(1,2)}=\Upsilon_{(2,1)}=I_{3 \times 3}$, and $\Upsilon_{(2,2)}=P^{-1}$. The matrix inequality $R \leq K_{R}$ is equivalent to $R^{-1} \geq K_{Z}$, where $K_{R}^{-1}=K_{Z}$.

In order to represent Eq. (22) and the matrix inequalities $\Sigma_{2} \geq 0$, and $\Upsilon>0$ in terms of LMIs, we pre- and post-multiply these matrix inequalities by $\operatorname{diag}\left(P^{-1}, P^{-1}\right), \operatorname{diag}\left(P^{-1}, P^{-1}\right)$, and $\operatorname{diag}\left(P^{-1}, I_{6 \times 6}\right)$, respectively, followed by applying the Schur complement to Eq.(22). We, then, substitute all the defined matrices of Eq.(14) in the resulting matrix inequalities. The nonlinear term $X Z^{-1} X$, which is obtained as a result of pre- and post- multiplying the last diagonal element of the matrix $\Sigma$ by $P^{-1}$ can be replaced by a linear lower bound as

$$
\begin{array}{r}
(Z-X)^{\mathrm{T}} Z^{-1}(Z-X)=Z-2 X+X Z^{-1} X \geq 0 \\
\Rightarrow X Z^{-1} X \geq 2 X-Z,
\end{array}
$$

Thus, we can conclude that LMIs stability conditions of Eqs.(12a), (12b), and (12c) in Theorem 1 are satisfied. In addition, to avoid large control gain matrices, which might be obtained by solving the LMI feasibility problem, we recall that $K=H P$. The control gain matrix $K$ can be upper-bounded by $K^{\mathrm{T}} K=P H^{\mathrm{T}} H P \leq k_{h} K_{P} K_{P}$, where $k_{h} \in \mathbb{R}>0$ corresponds to $H^{\mathrm{T}} H \leq k_{h}$. This expression is represented in the LMI form as in the first LMI stability condition of Eq.(12d) in Theorem 1. Furthermore, we recall that the effect of the system's nonlinearity appears in the terms $x^{\mathrm{T}} P x+\int_{t-\tau(t)}^{t} \int_{\eta}^{t}\left[f^{\mathrm{T}}(x(s)) R f(x(s))\right] \mathrm{d} s \mathrm{~d} \eta$ from the proposed LyapunovKrasovskii functional of Eq.(16). Thus, minimizing $P \leq K_{P}$ and $R \leq k_{R}$ can reduce the effect of the nonlinear term $f(x)$ and results in the larger estimate of the region of the attraction of the system which will be obtained throughout the next section. The term $R \leq K_{R}$ is represented in the LMI form as in the second LMI stability condition of Eq.(12d). Note that the term $P \leq K_{P}$ has been already expressed in the equivalent LMI form as in Eq.(12c). Therefore, by performing the LMI optimization problem in Theorem 1, the asymptotically stability of the nonlinear system of Eq.(7) is guaranteed at the origin, while the control gain matrices and the effect of the nonlinearity $f(x)$ are minimized.

It should be noted that in the proposed strategy for the purpose of designing the controller gain matrix $K$, a suitable Lyapunov-Krasovskii functional candidate is employed in the closed-loop dynamical system of Eq.(7) such that both $\gamma_{\max }$ and $\tau_{\max }$ are included in the proposed LMI. According to Theorem 1, to make the LMIs feasible and consequently the time derivative of 
the chosen Lyapunov-Krasovskii functional candidate negative definite, we assign a fixed value to $\tau_{\max }$ and choose a $\gamma_{\max }$, which makes the LMIs feasible. Then, we can conclude that the system is stable for $0 \leq \tau(t) \leq \tau_{\max }$ in the neighborhood $\mathscr{N}$ defined in Lemma 3. Likewise, if it is intended to find the maximum value of the time delay for a certain $\gamma_{\max }$ that the controlled system can tolerate then $\gamma_{\max }$ can be fixed and $\tau_{\max }$ is varied. The maximum estimate of the region of attraction can be then obtained in the next section by using $\gamma_{\max }$ to find a subset of the neighborhood $\mathscr{N}$.

\section{REGION OF ATTRACTION}

In the previous section, we showed that the time derivative of the Lyapunov-Krasovskii functional is negative in $\mathcal{C}\left(\left[-\tau_{\max }, 0\right], \mathscr{N}\right) \backslash(0,0)$, and therefore the linear delayed state feedback controller in Eq.(6) guarantees the asymptotic stability for the closed-loop dynamical system at the orgin. It should also be observed that the condition $\dot{V}\left(t, x_{t}(\theta)\right)<0$ does not necessary mean that any solution $x(t ; \phi)$ starting at the initial function $\phi(\theta),-\tau_{\max } \leq \theta \leq 0$ remains in the subset $\mathscr{N}$ for $t>0$. Indeed, we need to show that there is a region $R_{A}$ which is the subset of domain $\mathscr{N}$ such that $R_{A}=\{\phi(\theta) \subset \mathscr{N} \mid x(t ; \phi) \rightarrow 0$ as $t \rightarrow \infty\}$. The strategy is finding an estimate of the region of attraction by using the corresponding Lyapunov-Krasovskii functional expressed in Eq.(16). An estimate of the region of attraction can be considered as a compact positively invariant set $B_{r} \subset \mathscr{N}$ such that every trajectory starting in $B_{r}$ stays in $B_{r}$ for all future time [28]. For a given $r>0$ the compact set $B_{r}$ defined as

$$
B_{r}=\left\{x(t) \in \mathbb{R}^{6} \mid\|x(t)\| \leq r\right\} \subseteq \mathscr{N}
$$

In this section, we investigate the region of attraction of the closed-loop system resulting from the effect of the nonlinear term $f(x)$.

\section{A. Preliminary Results on Bounds of Lyapunov-Krasovskii functional}

Equation (16) is a bounded quadratic Lyapunov-Krasovskii functional of $x_{t}(\theta), \quad-\tau_{\max } \leq$ $\theta \leq 0$ such that $c_{1}\|x(t)\|_{\mathrm{av}}^{2} \leq V\left(t, x_{t}(\theta)\right)$ and for $t=0, V(\phi) \leq c_{2}\|\phi(\theta)\|_{m}^{2}$ where $\|\phi\|_{m}=$

$\sup \|\phi(\theta)\|,-\tau_{\max } \leq \theta \leq 0, \tau_{\max }\|x(t)\|_{\mathrm{av}}^{2}:=\int_{t-\tau}^{t}\|x(s)\|^{2} \mathrm{~d} s$, and $c_{1}$ and $c_{2}$ are constant positive scalars, respectively [26], [29]. With these two inequalities and the concept of the comparison 
method introduced in [28], Eq.(21) yields

$$
\dot{V}\left(t, x_{t}(\theta)\right) \leq \alpha\|x(t)\|^{2} \leq \alpha\|x(t)\|_{\mathrm{av}}^{2} \leq \frac{\alpha}{c_{1}} V\left(t, x_{t}(\theta)\right)
$$

where $\alpha=\lambda_{\max }(\Psi)<0$. Solving the above linear differential equation and considering the bounds of the quadratic Lyapunov Krasovskii functional defined above, we can obtain

$$
\begin{aligned}
& V\left(t, x_{t}(\theta)\right) \leq e^{\frac{\alpha}{c_{1}} t} V\left(x_{0}(\theta)\right)=e^{\frac{\alpha}{c_{1}} t} V(\phi(\theta)) \\
& \Rightarrow c_{1}\|x(t)\|_{\mathrm{av}}^{2} \leq V\left(t, x_{t}(\theta)\right) \leq e^{\frac{\alpha}{c_{1}}(t)} V(\phi(\theta)) \\
& \quad \leq c_{2} e^{\frac{\alpha}{c_{1}}(t)}\|\phi\|_{m}^{2}, \quad-\tau_{\max } \leq \theta \leq 0,
\end{aligned}
$$

Note that since $e^{\alpha t / c_{1}}$ is a monotonically decreasing function with an upper bound at $t=0$, inequality (26) can be relaxed to

$$
\|x(t)\|_{\mathrm{av}} \leq \sqrt{\frac{c_{2}}{c_{1}}}\|\phi\|_{m}
$$

Moreover, the above inequality should be valid inside the invariant set of $\|x(t)\|_{\mathrm{av}} \leq r$, so that using the definition of the compact set $B_{r}$ given by Eq.(25) results in

$$
\|x(t)\|_{\mathrm{av}} \leq \sqrt{\frac{c_{2}}{c_{1}}}\|\phi\|_{m} \leq r \Rightarrow\|\phi\| \leq\|\phi\|_{m} \leq r \sqrt{\frac{c_{1}}{c_{2}}} .
$$

This is a ball that can be considered as the region of attraction of the system. If the maximum value of the initial function starts inside this ball, the solution will remain inside this region and will go to the origin as $t \rightarrow \infty$. Note that the parameters $r, c_{1}, c_{2}$ which represent the radius of the compact set of $B_{r}$ and the lower bound and the upper bound of Lyapunov-Krasovskii functional, respectively, are still undetermined. The parameter $c_{1}$ using the Lyapunov-Krasovskii functional in Eq.(16) and the Jensen inequality [30] can be obtained as

$$
\begin{aligned}
& c_{1}\|x\|_{\mathrm{av}}^{2}=\left(\lambda_{\min }(P)+\tau_{\max } \lambda_{\min }(Q)\right. \\
& \left.+\tau_{\max }^{2} \lambda_{\min }(R)\right)\|x\|_{\mathrm{av}}^{2} \leq V\left(t, x_{t}(\theta)\right)
\end{aligned}
$$


For obtaining the constant term $c_{2}$, we find the upper bound of Eq.(16) as

$$
\begin{aligned}
& V(\phi)=x^{\mathrm{T}}(0) P x(0)+\int_{-\tau(t)}^{0} \phi^{\mathrm{T}}(s) Q \phi(s) \mathrm{d} s \\
& +\int_{-\tau(t)}^{0} \int_{\eta}^{0}\left[\phi^{\mathrm{T}}(s) A^{\mathrm{T}} R A \phi(s)+f^{\mathrm{T}}(s) R f(s)\right] \mathrm{d} s \mathrm{~d} \eta \\
& \leq\|P\|\|\phi(\theta)\|_{m}^{2}+\tau_{\text {max }}\|\phi(\theta)\|_{m}^{2}\|Q\|+\tau_{\text {max }}^{2}\|\phi(\theta)\|_{m}^{2} \\
& \left\|A^{\mathrm{T}} R A\right\|+\tau_{\text {max }}^{2} \gamma_{\text {max }}^{2}\|\phi(\theta)\|_{m}^{2}\|R\| \leq\left(\|P\|+\tau_{\text {max }}\|Q\|\right. \\
& \left.+\tau_{\text {max }}^{2}\left\|A^{\mathrm{T}} R A\right\|+\tau_{\text {max }}^{2} \gamma_{\text {max }}^{2}\|R\|\right)\|\phi(\theta)\|_{m}^{2} \\
& =c_{2}\|\phi(\theta)\|_{m}^{2},
\end{aligned}
$$

where we assumed that no control torque is implemented to the system over the initial time, i.e, $\theta \in\left[-\tau_{\max }, 0\right]$

\section{B. Region of Attraction for MRPs}

The proposed delayed feedback control law can generally guarantee the asymptotic stability of the origin of the minimal attitude coordinates that can satisfy the kinematics model of Eq.(1a). In this subsection, the set of MRPs are chosen, as an example of a minimal attitude representation which is extensively used in literature, and an estimate of the region of attraction of the system is obtained. Note that the unknown parameters $c_{1}$ and $c_{2}$ were obtained in the previous part. To find the last unknown parameter $r$ in Eq.(28), we obtain the upper bound of $f(x)$ in Eq.(4).

$$
\|f(x)\|^{2} \leq\left\|\mathfrak{G}\left(x_{1}\right)\right\|^{2}\left\|x_{2}\right\|^{2}+\beta^{2} \Lambda^{2}\left\|x_{2}\right\|^{4},
$$

where $\Lambda=\left\|J^{-1}\right\|\|J\|=\frac{\lambda_{\max }}{\lambda_{\min }} \geq 1$. Note that for spherical spacecraft $\Lambda=1$ and for other types of spacecraft $\Lambda>1$. The nonlinear function $\mathfrak{G}\left(x_{1}\right)$ in Eq.(1a) for the set of MRPs is defined as

$$
\mathfrak{G}\left(x_{1}(t)\right)=-x_{1}(t)^{\mathrm{T}} x_{1}(t) I_{3}+2 x_{1}(t)^{\times}+2 x_{1}(t) x_{1}^{\mathrm{T}}(t),
$$

with $\beta=4$. Thus The term $\left\|\mathfrak{G}\left(x_{1}\right)\right\|^{2}$ on the right hand side of Eq.(31) is calculated by using the definition of the induced 2-norm introduced in [28] as

$$
\begin{aligned}
& \left\|\mathfrak{G}\left(x_{1}\right)\right\|^{2}=\lambda_{\max }\left\{\left(\mathfrak{G}\left(x_{1}\right)^{\mathrm{T}}\left(\mathfrak{G}\left(x_{1}\right)\right)\right\}\right. \\
& =\lambda_{\max }\left\{\left(-x_{1}^{2} I_{3}-2 x_{1}^{\times}+2 x_{1} x_{1}^{\mathrm{T}}\right)\left(-x_{1}^{2} I_{3}+2 x_{1}^{\times}+2 x_{1} x_{1}^{\mathrm{T}}\right)\right\} \\
& =\lambda_{\max }\left\{x_{1}^{4} I_{3}-4 x_{1}^{2} I_{3} x_{1} x_{1}^{\mathrm{T}}-4 x_{1}^{\times^{2}}+4 x_{1} x_{1}^{\mathrm{T}} x_{1} x_{1}^{\mathrm{T}}\right\} \\
& =\lambda_{\max }\left\{x_{1}^{4} I_{3}-4 x_{1}^{\times 2}\right\},
\end{aligned}
$$


where $x_{1}^{2}=\left\|x_{1}\right\|^{2}=x_{1}^{\mathrm{T}} x_{1}$. By using the identity $x_{1}^{\times^{2}}=x_{1} x_{1}^{\mathrm{T}}-x_{1}^{2} I_{3}$ and the eigenvalues of $x_{1} x_{1}^{\mathrm{T}}$ obtained as $\left(x_{1}^{2}, 0,0\right)$, and substituting into the above mentioned equation, the maximum eigenvalue is given by

$$
\left\|\mathfrak{G}\left(x_{1}\right)\right\|^{2}=\left\|x_{1}\right\|^{2}\left(\left\|x_{1}\right\|^{2}+4\right) .
$$

We note that the same expression as in Eq.(32) is also obtained in [21] by using another approach. The following proposition can describe the upper bound of $x(t)$ where the set of MRPs are employed:

Proposition 1. The feedback system of Eq.(7) with the given $\gamma_{\max }$ satisfying Theorem 1 can guarantee the convergence of state variable $x(t)$ in a neighborhood of the origin with the radius given by

$$
r=\sqrt{\min \left\{\sigma_{u}, \frac{\gamma_{\max }^{2}}{16 \Lambda^{2}}\right\}}
$$

Proof. Substituting Eq.(32) into Eq.(31) and considering $\gamma_{\max }$ in the neighborhood $\mathscr{N}$ of origin introduced in Lemma 3, we can obtain

$$
\begin{aligned}
& \|f(x)\|^{2} \leq\left\|x_{2}\right\|^{2}\left(\left\|x_{1}\right\|^{2}\left(\left\|x_{1}\right\|^{2}+4\right)+16 \Lambda^{2}\left\|x_{2}\right\|^{2}\right) \\
& \leq \gamma^{2}(x(t))\|x\|^{2} \leq \gamma_{\text {max }}^{2}\left(\left\|x_{1}\right\|^{2}+\left\|x_{2}\right\|^{2}\right) .
\end{aligned}
$$

Therefore, a sufficient condition for Eq.(34) to hold can be obtained as

$$
\begin{array}{r}
16 \Lambda^{2}\left\|x_{2}\right\|^{4} \leq \gamma_{\text {max }}^{2}\left\|x_{2}\right\|^{2} \Rightarrow\left\|x_{2}\right\| \leq \frac{\gamma_{\max }}{4 \Lambda} \\
\left(\left\|x_{2}\right\|\left\|x_{1}\right\|\right)^{2}\left(\left\|x_{1}\right\|^{2}+4\right) \leq \gamma_{\max }^{2}\left\|x_{1}\right\|^{2} \\
\Rightarrow\left\|x_{1}\right\| \leq \sigma_{u}=\sqrt{16 \Lambda^{2}-4} .
\end{array}
$$

Note that $\sigma_{u} \geq 2 \sqrt{3}$ since $\Lambda>1$. To avoid switching to the shadow set at an arbitrary point $\|\sigma\| \leq \sigma_{0}$, where $\sigma_{0}$ is a positive constant scalar, due to the difficulty, which arises in designing a delayed feedback controller, $\sigma_{u}$ is chosen as $\sigma_{u}=\min \left\{\sigma_{0}, \sqrt{16 \Lambda^{2}-4}\right\}$. Equation (35) has been also obtained in [21]. However, switching surface $\|\sigma\|=\sigma_{0}$ has not been considered. Moreover, considering the compact set $\|x(t)\| \leq r$ we can obtain

$$
\|x(t)\|^{2}=\left\|x_{1}(t)\right\|^{2}+\left\|x_{2}(t)\right\|^{2} \leq \min \left\{\sigma_{u}^{2}, \frac{\gamma_{\max }^{2}}{16 \Lambda^{2}}\right\} .
$$

Consequently, $r$ can be obtained as Eq.(33) 
A conservative estimate of the region of attraction in terms of the initial function can be obtained from Eq.(28) as

$$
\begin{aligned}
& \|\phi(\theta)\| \leq \sqrt{\frac{c_{1}}{c_{2}} \min \left\{\sigma_{u}^{2}, \frac{\gamma_{\max }^{2}}{16 \Lambda^{2}}\right\}} \\
& \Rightarrow\left\|x_{1}(\theta)\right\|^{2}+\left\|x_{2}(\theta)\right\|^{2}=\|\sigma(\theta)\|^{2} \\
& +\frac{1}{16}\|\omega(\theta)\|^{2} \leq \sqrt{\frac{c_{1}}{c_{2}} \min \left\{\sigma_{u}^{2}, \frac{\gamma_{\max }^{2}}{16 \Lambda^{2}}\right\}}, \quad-\tau_{\max } \leq \theta \leq 0 .
\end{aligned}
$$

To ensure that there is no control input torque applied over the initial function and the state variables cannot escape from the estimated region of attraction, the upper bounds of the state variables in terms of the initial functions are obtained in [21] as

$$
\begin{aligned}
&\|\sigma(\theta)\| \leq \tan \left(\tan ^{-1}\left(\|\sigma\|_{m}\right)+\frac{\sqrt{\Lambda}}{4}\|\omega\|_{m} \tau_{\max }\right) \\
&\|\omega(\theta)\| \leq \sqrt{\Lambda}\|\omega\|_{m}, \quad-\tau_{\max } \leq \theta \leq 0 .
\end{aligned}
$$

Substituting the above inequalities in Eq.(37), we can obtain an estimate of the region of attraction of the system in terms of the initial value of MRPs and angular velocities at $t=0$ as

$$
\begin{aligned}
\tan ^{2} & {\left[\tan ^{-1}\left(\|\sigma\|_{m}\right)+\frac{\sqrt{\Lambda}}{4}\|\omega\|_{m} \tau_{\text {max }}\right] } \\
& +\frac{1}{16} \Lambda\|\omega\|_{m}^{2} \leq \frac{c_{1}}{c_{2}} \min \left\{\sigma_{u}^{2}, \frac{\gamma_{\text {max }}^{2}}{16 \Lambda^{2}}\right\} .
\end{aligned}
$$

\section{Simulation Results}

In this section, the proposed delayed feedback controller is implemented using the DDE integerator ddesd in Matlab and the Matlab convex programming package CVX version 2.1 with the cvx tolerance level chosen as cvx-precision('best'). Simulations are performed on a PC with the processor Intel(R) Core (TM) 2 Duo with the CPU clock time $3.14 \mathrm{GHz}$ The physical system parameters borrowed from [15], [16], [21] are given by $J=\operatorname{diag}(1000,500,700) \mathrm{kg} \cdot \mathrm{m}^{2}$. In this example, we assume that the spacecraft is subject to an unknown time-varying delay at time $t \geq t_{0}$ which without loss of generality, we assume that $t_{0}=0 \mathrm{~s}$. Therefore, the delayed feedback controller is switched at this time to stabilize the state variables of the system. The time-varying delay is assumed to be unknown to the controller; however for the purpose of the simulation we consider the time-varying delay in the form of $\tau(t)=\tau_{c}+d \sin (t)$ where $\tau_{c}$ represents a constant value. We set $d=0.02 \mathrm{~s}, \tau_{c}=0.5 \mathrm{~s}$, and 
a conservative upper bound for $\tau_{\max }$ is chosen as $\tau_{\max }=0.55 \mathrm{~s}$. This value of the time delay is much larger than what appears in [15], [21] that is $\tau_{\max }=0.0125 \mathrm{~s}$. In addition, this paper assumes the unknown time-varying delay, while in [15], [21], time delays are assumed to be constant. Parameter $\Lambda$ from the inertia matrix is given as $\Lambda=2$. Simulations are performed for two different minimal attitude coordinates, namely MRPs and exponential coordinates. Among the family of minimal attitude parameterizations, MRPs are prominent and are used extensively in the literature, while exponential coordinates provide the natural linearization of the configuration space $\mathrm{SO}(3)$ at the identity [4]. Exponential coordinates are obtained from the exponential map, which is a local diffeomorphism (non-singular) at the identity [22], [23]. In terms of principal rotation elements, they are defined as $x_{1}(t)=\Phi(t) \hat{e}$ with the corresponding nonlinear function $\mathfrak{G}\left(x_{1}\right)=\frac{1}{2} x_{1}^{\times}+\left(\frac{1}{\Phi^{2}}-\frac{1+\cos (\Phi)}{2 \Phi \sin (\Phi)}\right) x_{1}^{\times 2}$. Note that the exponential coordinates are actually nonsingular for $\Phi<\pi$. Nonsingularity of the exponential coordinates at $\Phi=0$ can be concluded by the power series expansion of $\sin (\Phi)$ and $\cos (\Phi)$ around the identity, which results in the zero order term $1 / 12$ at $\Phi=0$. Thus, throughout the numerical simulation, the magnitude of $\Phi$ is checked for being sufficiently small and a Taylor expansion to an arbitrary number of terms about $\Phi=0$ is replaced. Following the same procedure as we used to obtain an estimate of the region of attraction for MRPs, the estimate of the region of attraction in terms of the initial value of the exponential coordinates and angular velocities can be obtained as

$$
\begin{aligned}
& 18.53 \sinh ^{2}\left\{\sinh ^{-1}\left(0.23\|\Theta\|_{m}\right)+0.23 \sqrt{\Lambda}\|\omega\|_{m} \tau_{\max }\right\} \\
& +\Lambda\|\omega\|_{\tau}^{2} \leq \frac{c_{1}}{c_{2}} \min \left\{\pi^{2}, \frac{\gamma_{\max }^{2}}{\Lambda^{2}}\right\} .
\end{aligned}
$$

We apply Theorem 1 for a certain $\tau_{\max }$ and an initial guess of $\gamma_{\max }$ to check the stability condition of Theorem 1 and consequently obtain an estimated region of attraction. However, this estimated region can be maximized for both MRPs and exponential coordinates if terms $\frac{c_{1}}{c_{2}} \min \left\{\sigma_{u}=\sigma_{0}=1, \frac{\gamma_{\max }^{2}}{16 \Lambda^{2}}\right\}$ from Eq.(38) and $\frac{c_{1}}{c_{2}} \min \left\{\pi^{2}, \frac{\gamma_{\max }^{2}}{\Lambda^{2}}\right\}$ from the aboved-mentioned equation are maximized, respectively, for a range of $\gamma_{\max }$, which makes LMIs feasible. To do this, we set the aboved-mentioned terms as the cost function and employ the inbuilt MATLAB function fminsearch in conjunction with Theorem 1 to obtain suitable values for $\gamma_{\max }$ and $c_{1}, c_{2}$ (indirectly from Subsection IV-A) that results in the maximum estimate of the region of attraction.

The parameter $\gamma_{\max }$ that satisfies Eq.(1) and yields the maximum estimated region of attraction 
for the closed-loop dynamical system using MRPs is obtained as $\gamma_{\max }=18.5$. The constant control gain matrix $K$ is given by

$$
\begin{aligned}
& K=[\operatorname{diag}(-11.21,-5.22,-7.75) \\
&\operatorname{diag}(-444.41,-218.69,-308.18)] .
\end{aligned}
$$

Consequently, for the same system with the exponential coordinates, the parameter $\gamma_{\max }$ is obtained as $\gamma_{\max }=16.1$ and the control gain matrix is given by

$$
\begin{aligned}
K=[\operatorname{diag}(-4.08,-4.61,-4.60) \\
\\
\operatorname{diag}(-209.55,-153.98,-179.93)] .
\end{aligned}
$$

The region of attraction can be obtained using Eq.(38) for MRPs and Eq.(39) for the exponential coordinates. The estimated region of attraction for both attitude coordinates are shown in Fig(1). As seen in this figure, for the corresponding system with MRPs and the choice of the switching surface $\sigma_{0}=1$, the maximum region of attraction for a rest-to-rest maneuver, i.e, $\|\omega(\theta)\|=0$, in terms of the maximum allowed principal rotation angle is obtained as $\Phi_{\max }=120.01^{\circ}$. Thus, the obtained control gain matrix, when MRPs are employed, can stabilize the system for the initial attitudes up to $\Phi_{\max }=120.01^{\circ}$. It is worth mentioning that increasing $\sigma_{0}$ results in a larger estimated region of attraction. For example, choosing the switching surface $\sigma_{0}=2$, yields $\Phi_{\max }=156.94^{\circ}$ for the rest-to-rest maneuver. On the other hand, the estimated region of attraction for the exponential coordinates shows that the obtained control gain matrix is able to stabilize the system for the initial attitudes up to $\Phi=106.25^{\circ}$ which is smaller than the one obtained for MRPs.

The estimate of the region of the attraction for different types of spacecraft using MRPs are also depicted in Fig. 2. As seen in this figure when MRPs are employed a spherical spacecraft with three identical principal moments of inertia gives the largest estimated region of attraction.

To observe the response of the controlled system with the obtained control gain matrices for both MRPs and exponential coordinates, we assume that the spacecraft is initially at $\Phi=100^{\circ}$ and $\hat{e}=[-0.5320,0.7403,0.4110]^{\mathrm{T}}$ with the initial angular velocity $\omega(\theta)=$ $[0.2,0.04,-0.05]^{\mathrm{T}} \mathrm{rad} / \mathrm{s}$ for MRPs and $\Phi=80^{\circ}$ and the same principal rotation axis and the initial angular velocity as in MRPs for the exponential coordinates. The initial conditions in terms of MRPs and the exponential coordinates can be obtained as $\sigma(\theta)=[-0.2481,0.3452,0.1916]^{\mathrm{T}}$ 


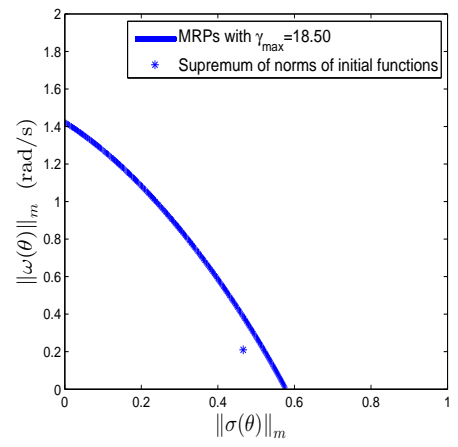

(a) Region of attraction

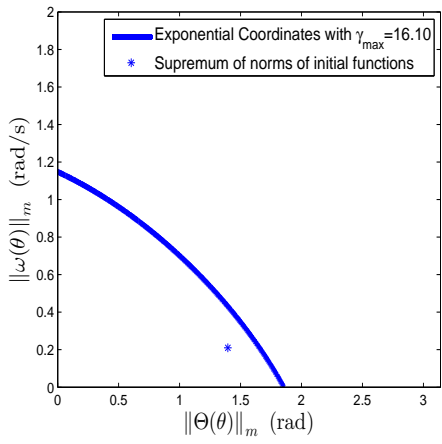

(b) Region of attraction

Fig. 1. (a) region of attraction when the set of MRPs are employed with (b) region of attraction when the exponential coordinates are employed with $\tau_{\max }=0.55 \mathrm{~s}, d=0.02 \mathrm{~s}$, and $\Lambda=2$.

and $\Theta(\theta)=[-0.7429,1.0337,0.5738]^{\mathrm{T}} \mathrm{rad}$, respectively (see.e.g. [5] for such a transformation). Figures $3 \mathrm{a}$ and $3 \mathrm{~b}$ show the controlled norms of the attitude coordinates for both MRPs and the exponential coordinates in terms of the principal rotation angle obtained by the proposed LMI approach. The controlled angular velocity and the magnitude of the control torque using both methods are also shown in Figs. 3c, 3d and 3e, 3f, respectively. Note that since we assumed that there is no control torque over the initial function, the controller starts working at $t=0.55 \mathrm{~s}$.

To demonstrate the significance of designing a suitable controller for the spacecraft with unknown time-varying time delay, let us assume that there is no time delay in the measurement and the spacecraft with MRPs is stabilized by a linear controller defined as $u(t)=K_{p} x(t)$, where $K_{p}=\left[-600 I_{3}-400 I_{3}\right]$ is the constant control gain matrix for the system without time delay. The spacecraft initial state variables are $\sigma(\theta)=[0,0.02,-0.02]^{\mathrm{T}}$ and $\omega(\theta)=[0.02,-0.01,0]^{\mathrm{T}} \mathrm{rad} / \mathrm{s}$. Then, at $t_{0}=0$, an unexpected unknown time delay in the measurement with $\tau_{\max }=0.55 \mathrm{~s}$ is implemented on the dynamical system. Figure 4 shows the effect of this time delay on the behavior of the controlled system. As seen in this figure, if we do not design a suitable controller for the delayed system and just use the control gain matrix $K_{p}$ which we used for the system without time delay, bad performance or even instability occurs for the state variables, but by using the proposed LMI approach and obtaining the suitable gain matrix the spacecraft can be perfectly stabilized in a reasonable time.

Table I compares the obtained region of attraction of the proposed control scheme in terms of the maximum principal rotation angle $\Phi_{\max }$ allowed for the rest-to-rest maneuver and a certain 

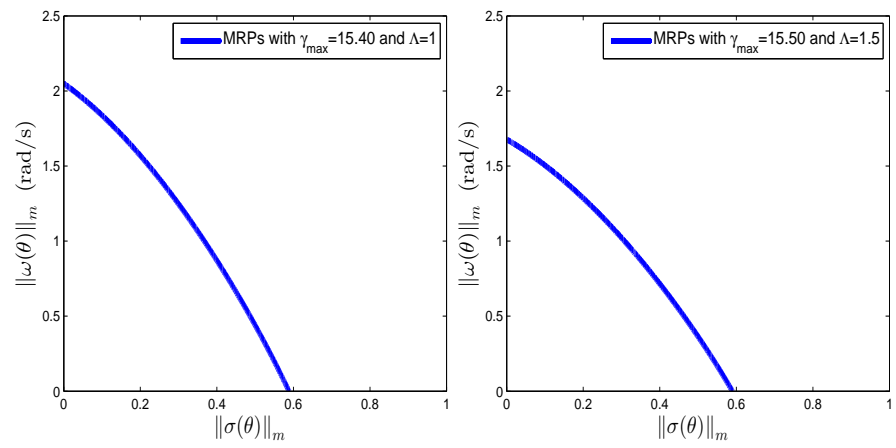

(a) $J_{1}=J_{2}=J_{3}=1000$

(b) $J_{1}=1500, J_{2}=J_{3}=1000$
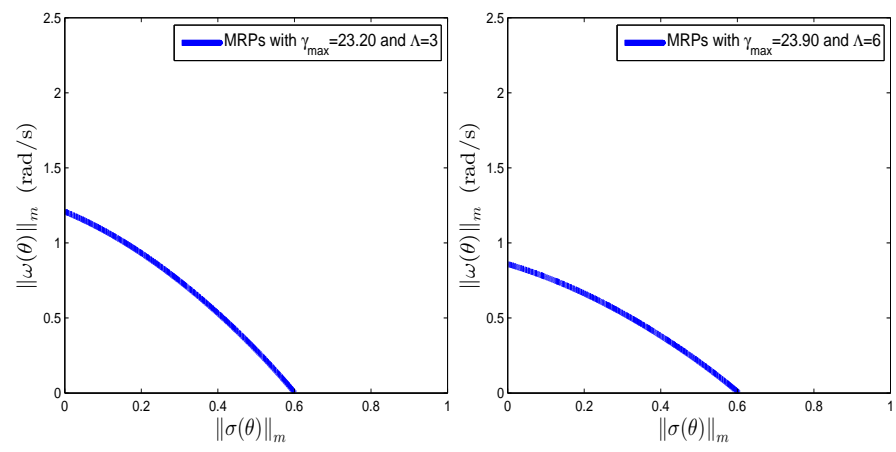

(c) $J_{1}=1500, J_{2}=J_{3}=500$

(d) $J_{1}=3000, J_{2}=J_{3}=500$

Fig. 2. An estimate of the region of attraction of the controlled system with MRPs for $\tau_{\max }=0.55 \mathrm{~s}$ and $d=0.02 \mathrm{~s}$.

time delay $\tau_{\max }$ with previous studies. As seen in this table, the size of the estimated region of attraction using the proposed method is much larger than other literature even when the system is subject to an unknown large time-varying delay with $\tau_{\max }=0.55 \mathrm{~s}$.

\section{CONCLUSION}

The delayed feedback control of spacecraft attitude dynamics has been studied where an unknown time-varying delay with a known upper bound was considered in the feedback path. The attitude representation was parameterized using a general class of minimal attitude coordinates. A linear continuous delayed state feedback controller was implemented to asymptotically stabilize the parameterized system. Using a suitable Lyapunov-Krasovskii functional in which a LMI was solved to obtain the controller gain matrix. Then, an estimate of the region of attraction of the nonlinear model was derived, inside which the asymptotic stability of parameterized system 


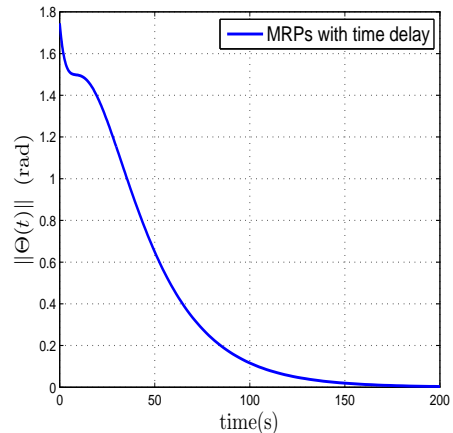

(a) attitude coordinate

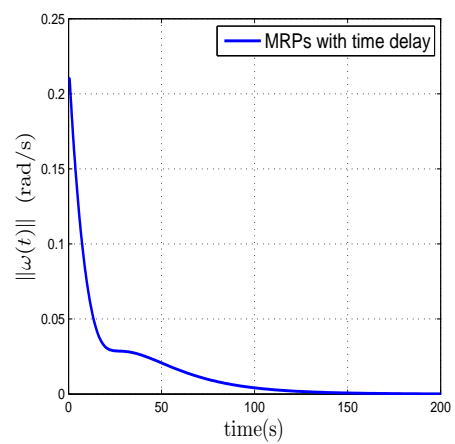

(c) angular velocity

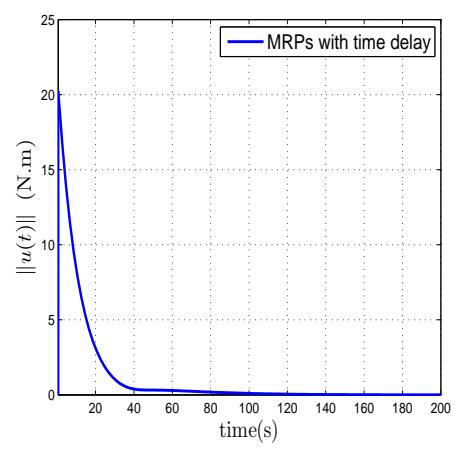

(e) control

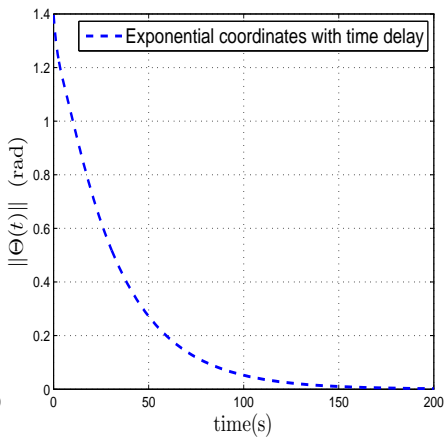

(b) attitude coordinate

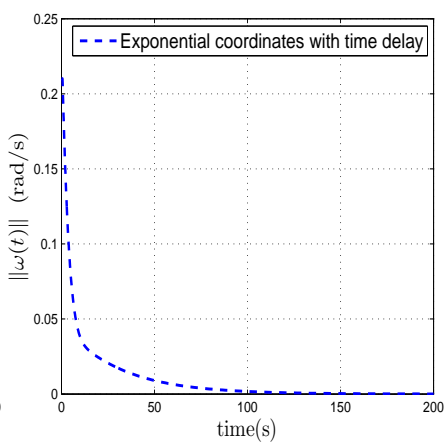

(d) angular velocity

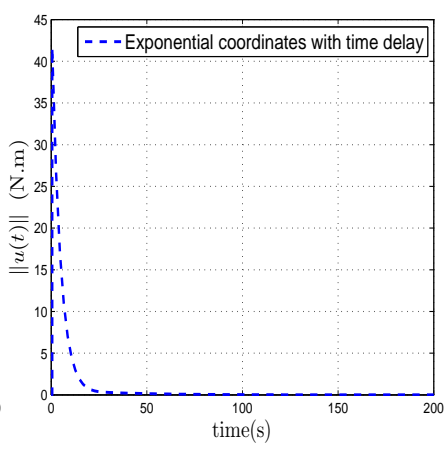

(f) control

Fig. 3. (a,c) controlled state trajectories and (e) control effort when the set of MRPs are employed with $\gamma_{\max }=18.5$ with the results obtained for $(b, d)$ controlled state trajectories and (f) control effort when the exponential coordinates are used with $\gamma_{\max }=16.1$ for $\tau_{\max }=0.55 \mathrm{~s}, d=0.02 \mathrm{~s}$, and $\Lambda=2$.

was guaranteed. The proposed controller was shown to be robust in presence of the unknown time-varying delay. 


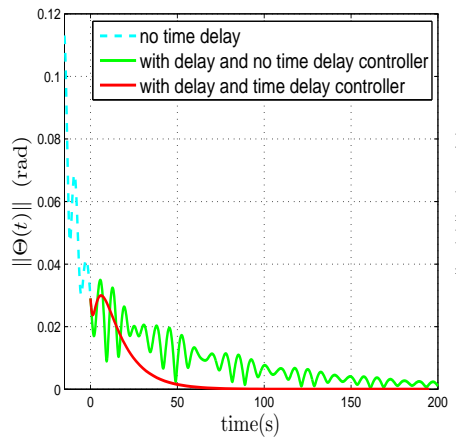

(a) attitude coordinate

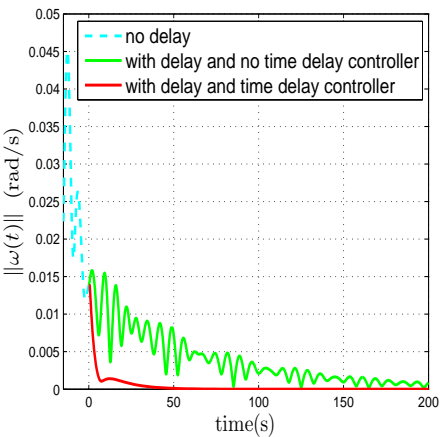

(b) angular velocity

Fig. 4. The effect of an unknown time varying delay on the performance of the stabilized spacecraft with the corresponding MRPs. (Dashed blue) the time before $t=0$ s shows the spacecraft without time delay controlled with a simple controller $u=K_{p} x(t)$ with an appropriate gain matrix $K_{p}=\left[-600 I_{6}-400 I_{6}\right]$. (Red solid) the time after $t=0$ shows the same spacecraft with an unknown time varying delay controlled with the suitable control gain matrix obtained using the proposed LMI approach for $\gamma_{\max }=10.4$ in comparison with (green solid) the same system with the control gain $K_{p}$ which fails to properly control the system in the presence of time delay at $t>0$.

\begin{tabular}{c|c|c|c}
\hline Ref & $\begin{array}{c}\text { Attitude } \\
\text { representation }\end{array}$ & $\begin{array}{c}\text { Control } \\
\text { structure }\end{array}$ & $\begin{array}{c}\text { Maximum } \phi \text { allowed } \\
\text { for certain } \tau_{\max }\end{array}$ \\
\hline$[15]$ & CRPs & nonlinear & $\begin{array}{c}\tau_{\max }=0.0125 \mathrm{~s} \\
\Phi_{\max }=0.0036 \mathrm{rad}\end{array}$ \\
\hline$[21]$ & MRPs & linear & $\begin{array}{c}\tau_{\max }=0.0125 \mathrm{~s} \\
\Phi_{\max }=0.0247 \mathrm{rad}\end{array}$ \\
\hline $\begin{array}{c}\text { The proposed } \\
\text { method }\end{array}$ & MRPs & linear & $\begin{array}{c}\tau_{\max }=0.55 \mathrm{~s} \\
\Phi_{\max }=2.0946 \mathrm{rad}\end{array}$ \\
\hline
\end{tabular}

TABLE I

A COMPARISON BETWEEN THE REGION OF ATTRACTION OBTAINED FROM DIFFERENT CONTROL STRATEGIES FOR THE ATTITUDE MOTION IN TERMS OF THE MAXIMUM PRINCIPAL ROTATION ANGLE $\Phi_{\max }$ FOR A REST-TO-REST MANEUVER AND A CERTAIN VALUE OF $\tau_{\max }$.

\section{ACKNOWLEDGMENT}

Financial support from the National Science Foundation under Grant No. CMMI-1131646 is gratefully acknowledged. 


\section{REFERENCES}

[1] H. Krishnan, M. Reyhanoglu, and H. McClamroch, "Attitude stabilization of a rigid spacecraft using two control torques: A nonlinear control approach based on the spacecraft attitude dynamics," Automatica, vol. 30, no. 6, pp. 1023-1027, 1994.

[2] F. Lizarralde and J. T. Wen, "Attitude control without angular velocity measurement: A passivity approach," Automatic Control, IEEE Transactions on, vol. 41, no. 3, pp. 468-472, 1996.

[3] P. Tsiotras, "Stabilization and optimality results for the attitude control problem," Journal of Guidance, Control, and Dynamics, vol. 19, no. 4, pp. 772-779, 1996.

[4] N. A. Chaturvedi, A. K. Sanyal, and N. H. McClamroch, "Rigid-body attitude control," Control Systems, IEEE, vol. 31, no. 3, pp. 30-51, 2011.

[5] H. Schaub and J. L. Junkins, Analytical Mechanics of Space Systems. Reston, VA: AIAA Education Series, 2009.

[6] H. Schaub, M. R. Akella, and J. L. Junkins, "Adaptive control of nonlinear attitude motions realizing linear closed loop dynamics," Journal of Guidance, Control, and Dynamics, vol. 24, no. 1, pp. 95-100, 2001.

[7] R. Sharma and A. Tewari, "Optimal nonlinear tracking of spacecraft attitude maneuvers," Control Systems Technology, IEEE Transactions on, vol. 12, no. 5, pp. 677-682, 2004.

[8] A. K. Sanyal and M. Chyba, "Robust feedback tracking of autonomous underwater vehicles with disturbance rejection," in American Control Conference, 2009. ACC'09. IEEE, 2009, pp. 3585-3590.

[9] J. Karpińska and K. Tchoń, "Optimal extended jacobian inverse kinematics algorithm with application to attitude control of robotic manipulators," Robot Motion and Control 2011, pp. 237-246, 2012.

[10] S. R. Marandi and V. J. Modi, "A preferred coordinate system and the associated orientation representation in attitude dynamics," Acta Astronautica, vol. 15, no. 11, pp. 833-843, 1987.

[11] M. D. Shucter, "A survey of attitude representations," Journal of the Astronautical Sciences, vol. 41, no. 4, pp. 439-517, 1993.

[12] H. Schaub and J. L. Junkins, "Stereographic orientation parameters for attitude dynamics: A generalization of the rodrigues parameters," Journal of the Astronautical Sciences, vol. 44, no. 1, pp. 1-19, 1996.

[13] T. Lee, "Geometric tracking control of the attitude dynamics of a rigid body on $\mathrm{SO}(3)$," in American Control Conference (ACC), 2011. IEEE, 2011, pp. $1200-1205$.

[14] A. K. Sanyal and N. A. Chaturvedi, "Almost global robust attitude tracking control of spacecraft in gravity," in AIAA Guidance, Navigation and Control Conference, Honolulu, HI, AIAA-2008-6979.

[15] A. Ailon, R. Segev, and S. Arogeti, "A simple velocity-free controller for attitude regulation of a spacecraft with delayed feedback," IEEE transactions on automatic control, vol. 49, no. 1, pp. 125-130, 2004.

[16] M. Sidi, Spacecraft Dynamics and Control: A Practical Engineering Approach. Cambridge: Cambridge University Press, 1997.

[17] M. Nazari, E. Samiei, E. Butcher, and H. Schaub, "Attitude stabilization using nonlinear delayed actuator control with an inverse dynamics approach,” AAS/AIAA Spaceflight Mechanics Meeting, 2012.

[18] M. Nazari, E. A. Butcher, and H. Schaub, "Spacecraft attitude stabilization using nonlinear delayed multiactuator control and inverse dynamics," Journal of Guidance, Control, and Dynamics, vol. 36, no. 5, pp. 1440-1452, 2013.

[19] E. Samiei, M. Nazari, E. Butcher, and H. Schaub, "Delayed feedback control of rigid body attitude using neural networks and lyapunov-krasovskii functionals," in AAS/AIAA Spaceflight Mechanics Meeting, Charleston, SC. UNIVELT INC, 2012, pp. 12-168. 
[20] J. Kim and J. Crassidis, "Robust spacecraft attitude control using model-error control synthesis," in AIAA Guidance, Navigation, and Control Conference, Monterey, CA, 2002.

[21] A. A. Chunodkar and M. R. Akella, "Attitude stabilization with unknown bounded delay in feedback control implementation," Journal of Guidance, Control, and Dynamics, vol. 34, no. 2, pp. 533-542, 2011.

[22] F. Bullo and R. M. Murray, "Proportional derivative (PD) control on the Euclidean group," vol. 2, Rome, Italy, Jun. 1995, pp. 1091-1097.

[23] F. Bullo and A. D. Lewis, Geometric Control of Mechanical Systems, ser. Texts in Applied Mathematics. New YorkHeidelberg-Berlin: Springer Verlag, 2004, vol. 49.

[24] M. P. Do Carmo, Riemannian geometry. Springer, 1992.

[25] E. Samiei, S. Torkamani, and E. A. Butcher, "On Lyapunov stability of scalar stochastic time-delayed systems," International Journal of Dynamics and Control, vol. 1, no. 1, pp. 1-17, 2013.

[26] K. Gu and S.-L. Niculescu, "Survey on recent results in the stability and control of time-delay systems," Journal of Dynamic Systems, Measurement, and Control, vol. 125, no. 2, pp. 158-165, 2003.

[27] Y. S. Moon, P. Park, W. Kwon, and Y. Lee, "Delay-dependent robust stabilization of uncertain state-delayed systems," International Journal of Control, vol. 74, no. 14, pp. 1447-1455, 2001.

[28] H. K. Khalil, Nonlinear Systems. New Jersey: Prentice Hall, 2002.

[29] K. Gu, J. Chen, and V. L. Kharitonov, Stability of time-delay systems. Springer, 2003.

[30] K. Gu, "An integral inequality in the stability problem of time-delay Systems," Proceedings of the 39th IEEE Conference on Decision and Control, pp. 2805-2810, 2000. 